\title{
Neutrophil Extracellular Trap Killing Assay of Candida albicans
}

Sheng-Yang Wu ${ }^{\$}$ and Betty A. Wu-Hsieh*

Graduate Institute of Immunology, National Taiwan University College of Medicine, Taipei, Taiwan; \$Present address: Institute of Molecular Medicine, National Taiwan University College of Medicine, Taipei, Taiwan

*For correspondence: bwh@ntu.edu.tw

\begin{abstract}
[Abstract] Fungal pathogen Candida albicans is one of the top leading causes of overall healthcareassociated bloodstream infections worldwide. Neutrophil is the major effector cell to clear C. albicans infection. Our study showed that mouse neutrophils utilize two independent mechanisms to kill $C$. albicans: one is CR3 downstream NADPH oxidase-dependent mechanism that kills opsonized $C$. albicans; the other one is dectin-2-mediated NADPH oxidase-independent neutrophil extracellular trap (NET) that kills unopsonized C. albicans. Neutrophil killing of opsonized C. albicans requires phagocytosing the organism and production of reactive oxygen species production (ROS). Most existing protocols that assay for neutrophil killing of $C$. albicans requires a washing step after allowing neutrophils to phagocytose the organism. By definition, NET kills organisms extracellularly. Therefore, it is important to skip the washing step and add an optimal ratio of neutrophils and C. albicans to the wells. To demonstrate the effect of NET, it is necessary to compare killing ability of neutrophils treated with micrococcal nuclease (MNase), an enzyme that digests NET, to that treated with heat-inactivated MNase. MNase is also applied to release NET-bound fungal elements for counting. This protocol can be applied to assay NET killing of other biofilm-forming organisms.
\end{abstract}

Keywords: Neutrophil extracellular trap, Candida albicans, Neutrophil, Fungicidal activity, Micrococcal nuclease

[Background] Candida albicans is an opportunistic fungal pathogen that resides as a commensal on mucosal surface and the skin in most humans. Environmental changes in temperature, nutrition, or the presence of serum induces its transformation from yeast form to hyphae. Candida infection is one of the top leading causes of overall healthcare-associated bloodstream infections in medical centers as well as regional hospitals. Mortality among patients with invasive candidiasis is as high as $40 \%$ even after receiving antifungal therapy (Brown et al., 2012; Chen et al., 2014; Kullberg and Arendrup, 2015). Patients with neutropenia and neutrophil dysfunction are at high risk for invasive candidiasis, suggesting the importance of neutrophil anti-Candida functions in host defense (Antachopoulos et al., 2007; Horn et al., 2009; Lionakis and Netea, 2013). Our work showed that mouse neutrophils utilize two independent mechanisms, one NADPH oxidase-dependent and the other NADPH oxidase-independent, to kill $C$. albicans. NADPH oxidase-dependent killing of opsonized $C$. albicans requires phagocytosis mediated by $\mathrm{CR} 3$, and NADPH oxidase-independent killing of unopsonized $C$ albicans is through NET formation mediated by dectin-2 (Wu et al., 2017 and 2019). MNase is an enzyme that digests NET. To demonstrate 
the effect of NET, it is necessary to compare the killing ability of neutrophils that is treated with MNase to that treated with heat-inactivated MNase. Difference between the two treatments denotes killing by NET and not by other factors (Wu et al., 2019). Neutrophils are phagocytic. Taking up microorganisms through receptors triggers robust ROS production that kills the engulfed organism. To assay for NADPH oxidase-dependent killing of opsonized C. albicans, one of the important steps is to wash off un-engulfed microorganisms after allowing time for phagocytosis to take place (Vonk et al., 2012; Wu et al., 2017). In contrast, since NET kills microorganisms extracellularly, it is critical not to wash off un-engulfed organisms to assay for NET-mediated killing of $C$. albicans. Moreover, instead of lysing neutrophils by $\mathrm{ddH}_{2} \mathrm{O}$ at high $\mathrm{pH}(\mathrm{pH} 11.0)$ to release ingested microorganisms, NET-forming neutrophils and fungal hyphal elements are detached from the wells by mini cell scraper and DNA digesting enzyme MNase (Wu et al., 2017 and 2019). The method described here were published in 2019 (Wu et al., 2019) to observe killing of $C$. albicans by NET. This method can be adapted to quantify NET-mediated killing of other biofilm-forming organisms.

\section{Materials and Reagents}

1. Pipette tips

2. Mini cell scraper (Biotium, catalog number: 22003)

3. Flat-bottomed 96-well plates (Corning, catalog number: 3599)

4. $15 \mathrm{ml}$ conical tube (Corning, catalog number: 430791)

5. $90 \mathrm{~mm} \times 15 \mathrm{~mm}$ Petri dish (Alpha Plus, catalog number: 16001)

6. $1.5 \mathrm{ml}$ microcentrifuge tube (Corning, catalog number: MCT-150-C)

7. $3 \mathrm{ml}$ syringe with 23-gauge needle (BD, catalog number: 302111$)$

8. Parafilm (Bemis, catalog number: PM996)

9. C. albicans SC5314 strain (ATCC MYA-2876)

10. Female inbred mice in C57BL/6JNarl background, 6-12 weeks of age (National Laboratory Animal Center, RMRC11005)

11. Percoll (GE Healthcare, catalog number: 17-0891-01)

12. 10x Dulbecco's Phosphate Buffered Saline (DPBS) (Biological Industries, catalog number: 02023-1A)

13. 10x Hank's Balanced Salt Solution (HBSS) (Biological Industries, catalog number: 02-015-5A)

14. $0.4 \%$ Trypan blue (Sigma-Aldrich, catalog number: 93595)

15. $7.5 \%$ sodium bicarbonate (Biological Industries, catalog number: 03-040-1B)

16. Yeast-peptone-dextrose (YPD) broth (Bioshop, catalog number: YPD002.500)

17. Agar A (BIO BASIC, catalog number: FB0010)

18. $2 \times 10^{6} \mathrm{U} / \mathrm{ml}$ Micrococcal nuclease (MNase) (NEB, catalog number: M0247S)

19. Sterile double distilled $\mathrm{H}_{2} \mathrm{O}\left(\mathrm{ddH}_{2} \mathrm{O}\right)$, adjusted to $\mathrm{pH} 11$ by $\mathrm{NaOH}$

20. 1x Dulbecco's Phosphate Buffered Saline (DPBS) (see Recipes)

21. 1x Hank's Balanced Salt Solution (HBSS) (see Recipes) 
22. YPD agar plate (see Recipes)

23. Red blood cell (RBC) lysis buffer (see Recipes)

24. $100 \%$ Percoll (see Recipes)

25. $55 \%, 62 \%$, and $81 \%$ Percoll (see Recipes)

26. Heat-inactivated micrococcal nuclease (MNase, see Recipes)

\section{Equipment}

1. Finnpipette ${ }^{\mathrm{TM}} \mathrm{F} 1$ Variable Volume Pipettes (Thermo Scientific, catalog numbers: $4641050 \mathrm{~N}$, $4641080 \mathrm{~N}$, and $4641100 \mathrm{~N}$ )

2. Counting chamber Neubauer-improved (HAUSSER, catalog number: CB73811-01490)

3. Autoclave Machine (see Note 2)

4. $37^{\circ} \mathrm{C}, 5 \% \mathrm{CO}_{2}$ incubator (see Note 2 )

5. $30{ }^{\circ} \mathrm{C}$ incubator for growing C. albicans yeasts (see Note 2)

6. Table top general-purpose centrifuge with swinging bucket rotor (KUBOTA, Model 4000, Rotor Name: ST-720M and PT-89M)

7. CellGard ES Energy Saver Class II Type A2 Biological Safety Cabinet (NuAire, model: NU-475400)

8. Vortex-Genie 2 (Scientific Industries)

9. Light Microscope (see Note 2)

10. Surgical operating dissecting scissors (sharp/sharp) (see Note 2)

11. Semken forceps (see Note 2)

12. Euthanasia chamber that is used to administer $\mathrm{CO}_{2}$ for euthanasia (see Note 2)

\section{Software}

1. Prism GraphPad Software (CA, USA)

\section{Procedure}

A. Mouse bone marrow neutrophil enrichment

1. Mice at 6-12 weeks of age are placed in euthanasia chamber and euthanized by carbon dioxide for 4 min according to the AVMA Guidelines on Euthanasia (Cima, 2013). Obtain femur and tibia by using surgical operating dissecting scissors and Semken forceps.

Note: All animal procedures and experimental protocols need to be approved by AAALACaccredited facility of the host institute.

2. Soak the bones in $1 \mathrm{ml}$ ice cold $1 \mathrm{x}$ HBSS.

3. Cut both ends of the bone, insert to one end the needle of a $3 \mathrm{ml}$ syringe that is filled with ice cold $1 x$ HBSS, push the plunger to flush out bone marrow cells into a $15 \mathrm{ml}$ conical tube. 
4. Flush the bone one more time as in Step A3.

5. Centrifuge the tube at $300 \mathrm{xg}$ in room temperature for $10 \mathrm{~min}$ and discard the supernatant.

6. Resuspend cell pellet in $1 \mathrm{ml}$ of ice cold RBC lysis buffer and leave it in room temperature for 1 $\min$.

7. Add $1 \mathrm{ml}$ of $1 \times$ DPBS to the tube.

8. Centrifuge the tube at $500 \times \mathrm{g}$ in room temperature for $5 \mathrm{~min}$, discard the supernatant and suspend the pellet in $1 \mathrm{ml}$ of $1 \times$ DPBS.

9. Prepare three layers of discontinuous Percoll solutions (55\%, $62 \%$, and $81 \%$ Percoll in the order of top to bottom in a $15 \mathrm{ml}$ conical tube. Each layer is consisted of $3 \mathrm{ml}$ as shown in Figure 1).

10. Overlay bone marrow cell suspension on top of the discontinuous Percoll gradient as shown in Figure 1.

11. Centrifuge at $1,400 \times g$ in room temperature for $30 \mathrm{~min}$ in a swing-out rotor, with the brake off.

12. "Gently" remove the top $6 \mathrm{ml}$ of the solution by micropipette and collect $1.0-1.5 \mathrm{ml}$ of the solution containing the band of neutrophils at the interface of 62 and $81 \%$ as shown in Figure 1 to a new $15 \mathrm{ml}$ conical tube.

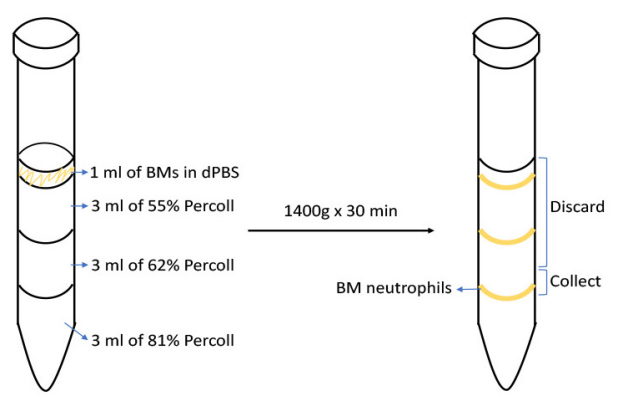

Figure 1. Enrichment of bone marrow neutrophils by discontinuous Percoll gradient centrifugation. Overlay bone marrow cell suspension on top of the 55/62/81\% Percoll gradient (Step A10). After centrifugation in swing-out rotor (Step A11), there will be three visible bands in the gradient solution. Neutrophils are located at the interface of $62 \%$ and $81 \%$ Percoll (the lowest band). Carefully remove the top $6 \mathrm{ml}$ of the solution and collect the top 1.0-1.5 ml of the remaining solution that contains cells at the lowest band (between the 2.5-4.0 mark on the scale) (Step A12).

13. Add $8 \mathrm{ml}$ of $1 \times$ DPBS to the tube containing neutrophils and centrifuge at $1,000 \times \mathrm{g}$ in room temperature for $5 \mathrm{~min}$ and discard the supernatant.

14. Suspend the pellet in $2 \mathrm{ml}$ of DPBS and centrifuge at $500 \times \mathrm{g}$ in room temperature for $3 \mathrm{~min}$ and discard the supernatant.

15. Suspend the pellet in $1 \mathrm{ml}$ of HBSS.

16. Dilute $10 \mu \mathrm{l}$ of well-mixed cell suspension in $390 \mu \mathrm{l}$ of $0.4 \%$ trypan blue solution and count the number of viable cells by loading $10 \mu$ l of the mixture to a Neubauer counting chamber. While viable cells are bright and shiny, dead cells stain blue. There is approximately $0.8 \times 10^{7}-1 \times 10^{7}$ 
bone marrow cells per mouse.

17. Leave cells in room temperature until use.

B. Preparation of fresh C. albicans

1. Thaw a frozen aliquot of $C$. albicans and plate it onto a YPD agar plate.

2. Incubate the dish at $30^{\circ} \mathrm{C}$ overnight.

3. Scrape $C$. albicans colonies with a sterile $1,000 \mu l$ tip and streak the yeasts on YPD agar plate.

4. Incubate the plate at $30^{\circ} \mathrm{C}$ overnight.

5. Scrape C. albicans colonies with a sterile $200 \mu \mathrm{l}$ tip and suspend in $1 \mathrm{ml} \mathrm{HBSS}$.

6. Take $10 \mu \mathrm{l}$ of well-mixed C. albicans solution, dilute it in $390 \mu \mathrm{l}$ of $4 \%$ trypan blue solution and count the number of viable yeast cells by loading $10 \mu \mathrm{l}$ of the mixture to a Neubauer counting chamber. While viable yeast cells are bright and shiny, dead cells stain blue (approximately 0.5 $\times 10^{7}-2 \times 10^{7}$ C. albicans yeasts per $\mathrm{ml}$ ).

7. Leave $C$. albicans on ice until use.

C. Neutrophils and C. albicans preparations

1. Dilute bone marrow neutrophils obtained from Step A16 to $2 \times 10^{5}$ per $1 \mathrm{ml}$ of HBSS solution.

2. Dilute freshly harvested $C$. albicans yeasts obtained from Step B7 to $4 \times 10^{5}$ per $1 \mathrm{ml}$ of HBSS.

3. Prepare $4 \times 10^{3} \mathrm{U} / \mathrm{ml}$ MNase by diluting $0.5 \mu \mathrm{l}$ of enzyme stock in $250 \mu \mathrm{l}$ of HBSS.

D. NET killing assay of $C$. albicans

1. Seed $2 \times 10^{4}$ neutrophils $(100 \mu \mathrm{l})$ in the wells of flat-bottomed 96-well plate and let adhere by incubation at $37^{\circ} \mathrm{C}\left(5 \% \mathrm{CO}_{2}\right.$ incubator) for $30 \mathrm{~min}$.

2. Leave wells for $100 \mu \mathrm{l}$ of HBSS without adding neutrophils as control as in Figure 2 .

3. Add $4 \times 10^{4}$ unopsonized $C$. albicans yeasts $(100 \mu \mathrm{l})$ to all the wells (including control well) as shown in Figure 2.

4. Add $0.5 \mu \mathrm{l}$ of MNase $\left(4 \times 10^{3} \mathrm{U} / \mathrm{ml}\right)$ at a final concentration of $10 \mathrm{U} / \mathrm{ml}$ or heat-inactivated MNase at otherwise equivalent concentration to appropriate wells as shown in Figure 2. Centrifuge the plate at $800 \times g$ in room temperature for $3 \mathrm{~min}$ to spin down the yeasts.

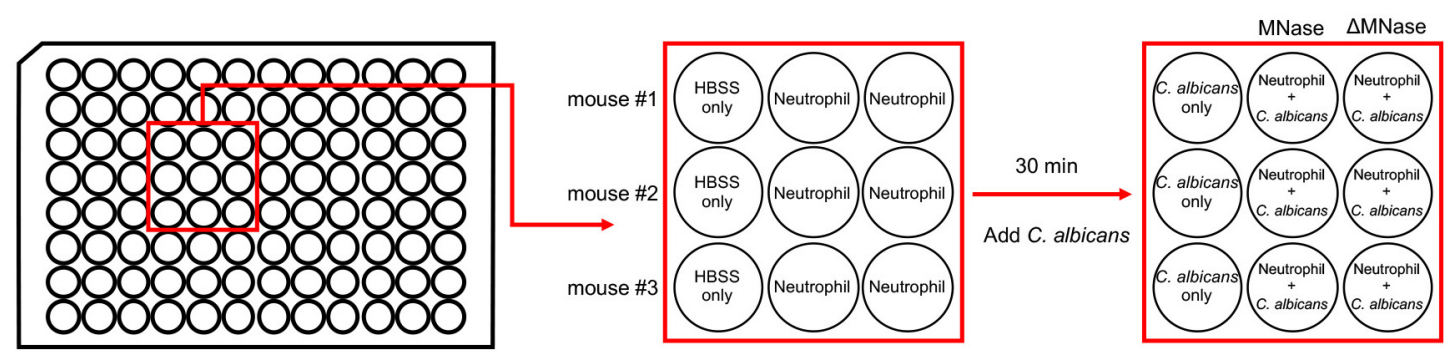

Figure 2. Layout for NET killing assay of $\boldsymbol{C}$. albicans. Bone marrow neutrophils obtained from three different mice are suspended in 1x HBSS separately. Neutrophils from each mouse are seeded in two separate wells (Step D1). Leave one well for 1x HBSS only (Step D2). After 
incubation of the plate at $37^{\circ} \mathrm{C}$ for $30 \mathrm{~min}, \mathrm{C}$. albicans suspensions are added to all three wells (Step D3). At the same time, MNase and heat-inactivated MNase ( $\triangle \mathrm{MNase})$ are added to separate wells separately (Step D4).

5. Incubate the plate at $37^{\circ} \mathrm{C}\left(5 \% \mathrm{CO}_{2}\right.$ incubator $)$ for $3 \mathrm{~h}$.

6. Add $0.5 \mu \mathrm{l}$ of MNase to all the wells.

7. Leave the plate at $37^{\circ} \mathrm{C}\left(5 \% \mathrm{CO}_{2}\right.$ incubator $)$ for $15 \mathrm{~min}$.

8. Collect the content (about $200 \mu \mathrm{l}$ ) of each individual well into a microcentrifuge tube.

9. Add $200 \mu \mathrm{l}$ of ice cold $\mathrm{H}_{2} \mathrm{O}(\mathrm{pH}=11)$ to each well.

10. Use mini cell scraper to scrape the bottom of each well followed by vigorous pipetting to dislodge C. albicans hyphae.

11. Collect the content of each well and add to the same microcentrifuge tubes.

12. Repeat steps 9 to 11 once (total of $600 \mu$ l solution in the microcentrifuge tube now).

13. Add $400 \mu \mathrm{l}$ of HBSS to each microcentrifuge tube.

14. Vortex microcentrifuge tubes vigorously for $20 \mathrm{~s}$.

15. Make 1:10 serial dilutions of the supernatant and plate $100 \mu \mathrm{l}$ of the diluted solution on YPD plate in duplicate.

16. Seal the plates with paraffin and leave in $30^{\circ} \mathrm{C}$ incubator for 2 days.

17. Enumerate colony forming units (CFUs) as shown in Figure 3.

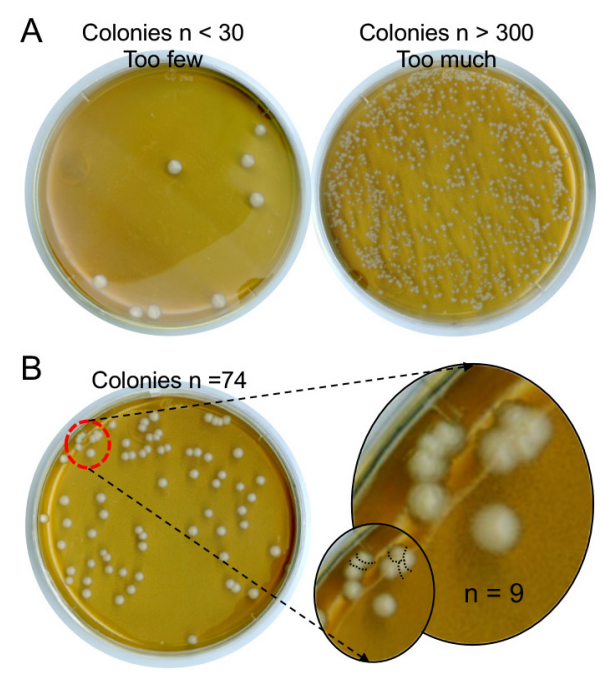

Figure 3. YPD plates with different $\boldsymbol{C}$. albicans colony forming units (CFUs). Supernatants containing C. albicans are serially diluted (Step D15) and plated on YPD plates. The plates are incubated at $30^{\circ} \mathrm{C}$ for 2 days (Step D16). C. albicans colonies are smooth and creamy white in color. There will be 3 different numbers of colonies from three 1:10 serially diluted supernatants. Select the plates with colonies fall between 30 and 300 (B) for counting and disregard the ones outside of this range (A). Carefully count each single colony even when they are very close to each other ( $B$, red dotted circle). 


\section{Data analysis}

1. CFU of control well is $\mathrm{N}_{0}$; CFU of wells containing MNase is $\mathrm{N}_{\mathrm{M}}$; CFU of wells containing heatinactivated MNase is $\mathrm{N}_{\triangle \mathrm{M}}$ as shown in Figure 4.

The average of CFU counts on the YPD dish in duplicate will be used as $\mathrm{N}_{0}, \mathrm{~N}_{M}$ and $\mathrm{N}_{\triangle \mathrm{M}}$.

2. $\%$ of killing in the MNase group $=\left(N_{0}-N_{M}\right) / N_{0} ; \%$ of killing in the heat-inactivated $M N a s e$ group $=\left(N_{0}-N_{\triangle M}\right) / N_{0}$ as shown in Figure 4.

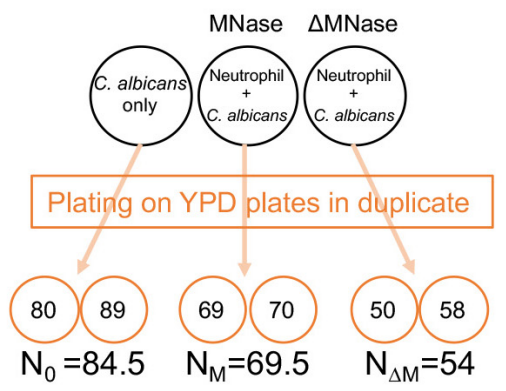

$\%$ of killing in MNase group $=(84.5-69.5) / 84.5=17.7 \%$

$\%$ of killing in $\Delta \mathrm{MNase}$ group $=(84.5-54) / 84.5=36.1 \%$

Figure 4. Calculation of \% NET killing of $C$. albicans. $N_{0}, N_{M}$ and $N_{\triangle M}$ are determined by the average of CFU counts of duplicate control wells (C. albicans only), wells containing neutrophils, C. albicans, and MNase and wells containing neutrophils, C. albicans, and heat-inactivated MNase, respectively. \% of killing is calculated as shown

3. To estimate the $\%$ of yeast cells killed by NET, data are analyzed by Mann-Whitney test by comparing the \% of killing in MNase group to that in heat-inactivated MNase group.

\section{Notes}

1. MNase digests NET. To include both MNase and inactivated MNase in Step D4 is to confirm that the killing is mediated by NET but not by other factors. To add MNase in Step D6 is to release NET-bound $C$. albicans from NET for subsequent plate count.

2. The reagents and materials from the manufacturers listed are those that have been used for this assay in the authors' laboratory. We have not tried reagents and materials from different manufacturers. The equipments that do not have their manufactures listed are common laboratory equipments.

3. It is important to completely lyse red blood cells because RBC contamination may interfere NET formation. One should also note that repeating or prolonging RBC lysis in Step A6 may result in neutrophil death. Therefore, if $1 \mathrm{ml}$ of ice cold RBC lysis buffer does not lyse all the RBCs, increase the volume but do not prolong the time for lysis.

4. Germination-defective strain of $C$. albicans (e.g., HLC 54 strain) does not induce NET formation (Wu et al., 2019). 
5. Colony counts on YPD plates between 30 and 300 ensures accurate counting. Plating of several 1:10 serial dilutions of the supernatants collected in microcentrifuge tubes will ensure that plating one of the dilutions will result in counts within the range.

\section{$\underline{\text { Recipes }}$}

1. 1x Dulbecco's Phosphate Buffered Saline (DPBS)

$5 \mathrm{ml}$ of $10 x$ DPBS is diluted in $45 \mathrm{ml}$ of $\mathrm{ddH}_{2} \mathrm{O}$

2. 1x Hanks' Balanced Salt Solution (HBSS)

$5 \mathrm{ml}$ of $10 \mathrm{x}$ HBSS is diluted in $44.64 \mathrm{ml}$ of $\mathrm{ddH}_{2} \mathrm{O}$ supplemented with $360 \mu \mathrm{l}$ of sodium bicarbonate

3. YPD agar plate
a. Add $50 \mathrm{~g}$ of YPD broth and $20 \mathrm{~g}$ of agar $\mathrm{A}$ into $1 \mathrm{~L}$ of $\mathrm{ddH}_{2} \mathrm{O}$
b. Stir the solution until YPD is dissolved
c. Cool to $50^{\circ} \mathrm{C}$ after autoclaving, and pour $23 \mathrm{ml}$ of the medium into sterile $10-\mathrm{cm}$ Petri dishes
d. Store YPD dishes in refrigerator until use

4. RBC lysis buffer
a. $\quad 0.61 \mathrm{~g}$ of Tris- $\mathrm{HCl}$ and $4.15 \mathrm{~g}$ of $\mathrm{NH}_{4} \mathrm{Cl}$ are dissolved in $500 \mathrm{ml}$ of $\mathrm{ddH}_{2} \mathrm{O}$
b. Adjust the $\mathrm{pH}$ value to 7.4 and autoclave

5. $100 \%$ Percoll

Mix $45 \mathrm{ml}$ of Percoll stock with $5 \mathrm{ml}$ of 10x DPBS

6. $55 \%, 62 \%$, and $81 \%$ Percoll

$55 \%, 62 \%$, and $81 \%$ Percoll are prepared by diluting $100 \%$ Percoll (prepared in Recipe 5) with $1 \times$ DPBS

7. Heat-inactivated MNase

Heat MNase $\left(4 \times 10^{3} \mathrm{U} / \mathrm{ml}\right)$ at $65^{\circ} \mathrm{C}$ for $4 \mathrm{~h}$ and store in refrigerator until used

\section{Acknowledgments}

This work was supported by Academia Sinica thematic project AS-105-TP-B08 to BWH and the Ministry of Science and Technology research grants 104-2320-B-002-052-MY2 and 107-2321-B002-053-MY3 to BWH and SYW, respectively. This protocol was adapted from a publication by Vonk et al. (2012) and modified according to protocols that quantified the viability of biofilm-forming microorganisms in other studies ( Morici et al., 2016; Mohammed et al., 2017).

\section{Competing interests}

NO financial competing interests. 


\section{Ethics}

Mouse study was carried out in strict accordance with the recommendations in the Guidebook for the Care and Use of Laboratory Animals, The Third Edition published by The Chinese-Taipei Society of Laboratory Animal Sciences in 2007. All animal procedures and experimental protocols were approved by AAALAC-accredited facility, the Committee on the Ethics of Animal Experiments of the National Taiwan University College of Medicine (Permit Number: 20140304, 20140533 and 20180013).

\section{References}

1. Antachopoulos, C., Walsh, T. J. and Roilides, E. (2007). Fungal infections in primary immunodeficiencies. Eur J Pediatr 166(11): 1099-1117.

2. Brown, G. D., Denning, D. W., Gow, N. A., Levitz, S. M., Netea, M. G. and White, T. C. (2012). Hidden killers: human fungal infections. Sci Trans/ Med 4(165): 165rv113.

3. Chen, P. Y., Chuang, Y. C., Wang, J. T., Sheng, W. H., Yu, C. J., Chu, C. C., Hsueh, P. R., Chang, S. C. and Chen, Y. C. (2014). Comparison of epidemiology and treatment outcome of patients with candidemia at a teaching hospital in Northern Taiwan, in 2002 and 2010. J Microbiol Immunol Infect 47(2): 95-103.

4. Cima, G. (2013). AVMA Guidelines for the Euthanasia of Animal: 2013 Edition. Javma-J Am Vet Med A 242: 715-716.

5. Horn, D. L., Neofytos, D., Anaissie, E. J., Fishman, J. A., Steinbach, W. J., Olyaei, A. J., Marr, K. A., Pfaller, M. A., Chang, C. H. and Webster, K. M. (2009). Epidemiology and outcomes of candidemia in 2019 patients: data from the prospective antifungal therapy alliance registry. Clin Infect Dis 48(12): 1695-1703.

6. Kullberg, B. J. and Arendrup, M. C. (2015). Invasive Candidiasis. N Engl J Med 373(15): 14451456.

7. Lionakis, M. S. and Netea, M. G. (2013). Candida and host determinants of susceptibility to invasive candidiasis. PLoS Pathog 9(1): e1003079.

8. Mohammed, M. M. A., Pettersen, V. K., Nerland, A. H., Wiker, H. G. and Bakken, V. (2017). Quantitative proteomic analysis of extracellular matrix extracted from mono- and dual-species biofilms of Fusobacterium nucleatum and Porphyromonas gingivalis. Anaerobe 44: 133-142.

9. Morici, P., Fais, R., Rizzato, C., Tavanti, A. and Lupetti, A. (2016). Inhibition of Candida albicans biofilm formation by the synthetic lactoferricin derived Peptide hLF1-11. PLoS One 11(11): e0167470.

10. Vonk, A. G., Netea, M. G. and Kullberg, B. J. (2012). Phagocytosis and intracellular killing of Candida albicans by murine polymorphonuclear neutrophils. Methods Mol Biol 845: 277-287. 
11. Wu, S. Y., Huang, J. H., Chen, W. Y., Chan, Y. C., Lin, C. H., Chen, Y. C., Liu, F. T. and WuHsieh, B. A. (2017). Cell intrinsic Galectin-3 attenuates neutrophil ROS-dependent killing of Candida by modulating CR3 downstream Syk activation. Front Immunol 8: 48.

12. Wu, S. Y., Weng, C. L., Jheng, M. J., Kan, H. W., Hsieh, S. T., Liu, F. T. and Wu-Hsieh, B. A. (2019). Candida albicans triggers NADPH oxidase-independent neutrophil extracellular traps through dectin-2. PLoS Pathog 15(11): e1008096. 